\title{
Enhancement of the Dissolution Rate of Indomethacin by Solid Dispersions in Low-substituted Hydroxypropyl Cellulose
}

\author{
ESTEFANÍA TASCÓN-OTERO, PALOMA TORRE-IGLESIAS ${ }^{1 *}$, J. J. GARCÍA-RODRÍGUEZ², M. ÁNGELES PEÑA AND \\ COVADONGA ÁLVAREZ-ÁLVAREZ ${ }^{1}$
}

Department of Pharmaceutics and Food Technology, Faculty of Pharmacy, ${ }^{1}$ Institute of Industrial Pharmacy, ${ }^{2}$ Department of Microbiology and Parasitology, Faculty of Pharmacy, Complutense University, 28040 Madrid, ${ }^{3}$ Department of Biomedical Science University of Alcalá, Ctra. Madrid-Barcelona Km. 33.6, 28871 Alcalá de Henares, Spain

Tascón-Otero et al.: Enhancement of Dissolution Rate of Indomethacin Solid Dispersions

\begin{abstract}
In the present study, new indomethacin formulations were developed in order to enhance the indomethacin dissolution rate by preparing solid dispersions using the freeze-drying method. The degree of alterations in the crystallinity of indomethacin was assessed according to the preparation method and by the addition of low-substituted hydroxypropyl cellulose. Solid dispersions improved the dissolution rate of indomethacin. Combined use of scanning electron microscopy, X-ray powder diffraction and differential scanning calorimetry revealed the basis of the increase in dissolution rate of indomethacin when formulated as lowsubstituted hydroxypropyl cellulose solid dispersions.
\end{abstract}

Key words: Indomethacin, solid dispersions, low-substituted hydroxypropyl cellulose, freeze-drying

Indomethacin (IND) is a non-steroidal antiinflammatory agent with antiinflammatory, analgesic and antipyretic activity. Its pharmacological effect is thought to be mediated through the inhibition of the enzyme cyclooxygenase (COX), which is responsible for catalysing the rate-limiting step in prostaglandin synthesis ${ }^{[1,2]}$. However, IND is relatively versatile, and further studies have pointed to the potential use in cancer prevention therapy. Some studies have reported activity on melanoma and non-melanoma skin cancers in animal and in vitro studies ${ }^{[3]}$. Clinical studies have found that topical or oral non-steroidal antiinflammatory drugs may lead to the regression of skin neoplasms ${ }^{[4]}$. A recent work reported that prostaglandin E2 (PGE2) is secreted by resistant tumour cells and inhibition of PGE2 production by IND eliminated the macrophage suppression factor from the supernatant and sensitized resistant tumour cells to macrophage cytotoxicity ${ }^{[5]}$. This study emphasized the important role of tumoursecreted PGE2 in escaping macrophage surveillance, and justifies the use of COX inhibitors as an adjuvant for improving tumour immunotherapy.

IND is classified as class II due to its poor solubility and high permeability ${ }^{[6]}$. According to the Biopharmaceutics

*Address for correspondence

E-mail: pmtorre@ucm.es

September-October 2019
Classification System, a drug compound is poorly soluble if the highest dose strength is not soluble in $250 \mathrm{ml}$ aqueous media over the $\mathrm{pH}$ range at $37^{\circ[7]}$. Over $40 \%$ of active pharmaceutical ingredients in development pipelines are poorly water-soluble drugs, which limit the formulation approaches and their clinical application and marketability due to their low dissolution and bioavailability ${ }^{[8]}$.

Various formulation techniques have been developed to enhance the water solubility of IND, such as salt formation $^{[9]}$, microemulsions ${ }^{[10]}$, nanosuspensions ${ }^{[11]}$, polymeric micelles ${ }^{[12]}$, nanoemulsions ${ }^{[13]}$, microspheres $^{[14]}$, particle size reduction ${ }^{[15]}$, cyclodextrins $^{[16]}$, prodrug formation ${ }^{[17]}$, solid-lipid nanoparticle ${ }^{[18]}$ and solid dispersion, which is considered one of the most successful strategies to improve the dissolution profile of poorly soluble drugs. The term solid dispersion refers to the dispersion of one or more

This is an open access article distributed under the terms of the Creative Commons Attribution-NonCommercial-ShareAlike 3.0 License, which allows others to remix, tweak, and build upon the work non-commercially, as long as the author is credited and the new creations are licensed under the identical terms

Accepted 22 July 2019

Revised 11 April 2019

Received 26 November 2018

Indian J Pharm Sci 2019;81(5):824-833 
active ingredients in an inert carrier or matrix in the solid state ${ }^{[19]}$. Low-substituted hydroxypropylcellulose $(\mathrm{L}-\mathrm{HPC})^{[20]}$ and sodium dodecyl sulphate (SDS) ${ }^{[21]}$ have been used by several researchers as carriers to obtain solid dispersions of poorly soluble drugs. L-HPC, as an inert carrier, has properties as a disintegrant ${ }^{[22]}$, and also improves the wettability and dispersibility of solid dispersions, which may influence the drug's dissolution behaviour ${ }^{[23-25]}$. SDS is an anionic surfactant that enhances drug wetting ${ }^{[21]}$. The objective of the present study was to evaluate the feasibility of L-HPC in altering the crystalline property of IND and enhancing its dissolution rate by preparing solid dispersions using a freeze-drying method.

\section{MATERIALS AND METHODS}

IND was supplied by Fagron Iberica SAU, Spain. SDS was supplied by Fischer Scientific, England. L-HPC was supplied by Shin-Etsu ${ }^{\circledR}$, Tokyo, Japan. All other chemical reagents were of pharmaceutical grade or higher.

\section{Preparation of formulations:}

IND solid dispersions were prepared using the freezedrying method using L-HPC as a carrier ${ }^{[26]}$. Solid dispersions were prepared with ratios of IND-SDSL-HPC, 1:0.06:0; 1:0.12:0; 1:0.12:5; 1:0.12:10 and 1:0.12:20 (w/w/w). The required amounts of IND and L-HPC were co-dissolved in $20 \mathrm{ml}$ of solution with $0.125 \mathrm{mg} / \mathrm{ml}$ of SDS, except for SD-1:0.06:0, which was co-dissolved in $20 \mathrm{ml}$ of solution with of $0.075 \mathrm{mg} / \mathrm{ml}$ of SDS, in a $\mathrm{pH} 8.4$ buffer (prepared as described in the United States Pharmacopeia). The samples were then frozen at $-40^{\circ}$ for at least $24 \mathrm{~h}$ and freeze-dried using a Liolabar 7 (Telstat Inc., Madrid, Spain). They were loaded on the freeze-dryer shelf at $5^{\circ}$. After thermal equilibration, the shelf temperature was lowered to $-40^{\circ}$ and the product was maintained at this temperature for $2 \mathrm{~h}$. The system was then evacuated to a pressure of 0.04 mbar and the shelf temperature was adjusted to $-35^{\circ}$ and maintained for $4 \mathrm{~h}$, before being raised to $-20^{\circ}(2 \mathrm{~h})$. After the freeze-drying process, each formulation was ground and sieved to obtain a particle size fraction of $0.125-0.500 \mathrm{~mm}^{[26]}$; the vials were capped within $5 \mathrm{~min}$ and stored at room temperature $\left(22-24^{\circ}\right)$ in a desiccator containing silica gel. The physical mixture (PM) containing an INDSDS-L-HPC ratio of 1:0.12:20 (w/w/w) was prepared by manually mixing the appropriate amount of IND with particle size fractions of $0.125-0.500 \mathrm{~mm}$ and carrier in a ceramic bowl using a polymeric spatula. SD-0:0.12:0 was obtained by the same preparation method as for the solid dispersions but without IND.

\section{Wetting property measurement:}

The contact angles on the powder compacts were measured on a Dataphysics OCA contact angle system equipped with a conventional goniometer and a highperformance video camera controlled by the OCA20 software provided by the manufacturer. A syringe connected to a Teflon capillary with an inner diameter of approximately $2 \mathrm{~mm}$ was used to supply liquid from above to measure the drops. Briefly, $200 \mathrm{mg}$ of sample was weighed and compressed using a circular stainlesssteel punch and die assembly (diameter $1 \mathrm{~cm}$ ) in an infrared tablet press (dwell time of $30 \mathrm{~s}$ and pressure of 8 tons). A drop of deionized water $(2.5 \mu \mathrm{l})$ was placed on the compressed plate and the measurement of the contact angle was initiated in the dynamic mode. The camera captures images with a frame size of $640 \times 480$ pixels at a maximum rate of 60 frames per second. The images are analysed using the axisymmetric drop shape analysis profile (ADSA-P) to obtain the contact length and height of the droplet. Finally, these parameters are used to estimate the contact angle of the droplet. At least two measurements were made per sample to ensure reproducibility. All the measurements were made at room temperature.

\section{In vitro dissolution studies:}

Dissolution assays of IND powder, SD-1:0.06:0, SD1:0.12:0, SD-1:0.12:5, SD-1:0.12:10, SD-1:0.12:20 and PM-1:0.12:20 were carried out using the DT80 dissolution system (Erweka GMBH, Germany). Initially, the dissolution rate of different formulations was studied in simulated gastric fluid without enzyme $(0.1 \mathrm{~N} \mathrm{HCl})$, and the dissolution data showed low drug release (less than $12.7 \%$ ). Other authors ${ }^{[20]}$ have reported that less than $6.2 \%$ of IND was dissolved at $\mathrm{pH} 1.2$, hence phosphate buffer of $\mathrm{pH} 6.8$ was used as a dissolution medium in this method. Other authors have achieved satisfactory results at a similar $\mathrm{pH}^{[27]}$. However, at $\mathrm{pH} 6.8$ the drug dissolves so rapidly that it is impossible to observe differences between the dissolution profiles of the various solid dispersions. Dissolution assays were therefore also performed at $\mathrm{pH}$ 4.5 in order to obtain slower dissolution profiles and allow the observation of differences in the dissolution rates between the different formulations. A USP apparatus 2 (paddle) was set up with a rotational speed 
of $50 \mathrm{rpm}$ and $900 \mathrm{ml}$ of dissolution medium. The temperature was maintained at $37.0 \pm 0.1^{\circ}$ throughout the dissolution study. An amount of solid dispersion equivalent to $10 \mathrm{mg}$ of IND was introduced into the vessel. At predetermined times, a $5 \mathrm{ml}$ sample was withdrawn and filtered through a $0.45 \mu \mathrm{m}$ filter $\left(\right.$ Minisart $\left.^{\mathbb{}}\right)$. The quantity of IND was determined at $266 \mathrm{~nm}$ using a UV/Vis 1700 spectrophotometer (Shimadzu, Japan).

As the carrier L-HPC, SDS, and the solvent components $\left(\mathrm{NaOH}, \mathrm{H}_{3} \mathrm{BO}_{3}\right.$ and $\left.\mathrm{KCl}\right)$ do not absorb $\mathrm{UV}$, these do not interfere at the chosen wavelength. The cumulative amount of IND released from the system was determined from the following calibration curves at $\mathrm{pH} 1.2,6.8$ and 4.5, respectively, $\mathrm{y}=0.0517 \mathrm{x}+0.0068$ $\left(\mathrm{r}^{2}=0.9999\right), \quad \mathrm{y}=0.0526 \mathrm{x}+0.0166 \quad\left(\mathrm{r}^{2}=0.9993\right) \quad$ and $\mathrm{y}=0.0587 \mathrm{x}+0.0027 \quad\left(\mathrm{r}^{2}=0.9995\right)$. The results obtained from the dissolution assays of different formulations were analysed using one-way analysis of variance (ANOVA), followed by Fisher's least statistical difference for multiple comparisons. A p-value of less than 0.05 was considered significant.

\section{Scanning electron microscopy (SEM) studies:}

IND powder, SD-0:0.12:0, SD-1:0.12:0, SD-1:0.12:5, SD-1:0.12:10, SD-1:0.12:20, PM-1:0.12:20 and L-HPC were placed on an aluminium sample mount. After coating with a thin layer of gold-palladium, the samples were analysed with a Jeol ${ }^{\circledR} 6400$ SEM. All the micrographs were produced by secondary electron imaging used for surface morphology identification at magnifications of $500 \times$ and an accelerating voltage of $20 \mathrm{kV}$.

\section{X-ray powder diffraction (XRPD): crystal structure characterization:}

The structure of the material was characterized by means of conventional $\theta-2 \theta$ powder X-ray diffraction with $\mathrm{Cu}-\mathrm{k} \alpha$ radiation (Philips X'Pert-MPD) in several of the samples (IND powder, SD-1:0.12:0, SD1:0.12:10, SD-1:0.12:20, PM-1:0.12:20 and L-HPC) in the study at room temperature. The $5-35^{\circ} 2 \theta$ range was scanned at a $0.04^{\circ}$ step size and $1 \mathrm{~s}$ time per step in all cases. Approximately $20 \mathrm{mg}$ of sample was dispersed on a zero-background Si sample holder, taking care was not to introduce a preferential orientation of the crystals.

\section{Differential scanning calorimetry (DSC):}

The thermal behaviour of the pure IND; SD-1:0.12:0,
SD-1:0.12:10, SD-1:0.12:20, PM-1:0.12:20 and L-HPC was characterized by means of DSC analysis. DSC thermograms were obtained with an automatic thermal analyser system (Mettler Toledo TC15, TA controller). Temperature was calibrated using the indium calibration reference standard (transition point: $\left.156.60^{\circ}\right)$. Samples of about $2 \mathrm{mg}$ were accurately weighed into aluminium pans, then hermetically sealed with aluminium lids and heated from 30 to $220^{\circ}$ at a heating rate of $10^{\circ} / \mathrm{min}$ under constant purging of dry nitrogen for $20 \mathrm{ml} / \mathrm{min}$. An empty pan, sealed in the same way as the sample, was used as a reference.

\section{RESULTS AND DISCUSSION}

The deionized water contact angle was measured on SD tablets (SD-1:0.12:0; SD-1:0.12:5 and SD-1:0.12:20), a PM tablet (PM-1:0.12:20) and a tablet of compacted pure drug. Surface roughness and potential polymer swelling are known to potentially influence wetting behaviour ${ }^{[28]}$. As can be seen in fig. 1, the water contact angle on the tablets gradually decreased between $1 \mathrm{~s}$ and $4 \mathrm{~s}$. The IND tablet had the highest water contact angle $\left(107 \pm 2^{\circ}\right.$ after $\left.1 \mathrm{~s}\right)$, which was attributed to the stronger hydrophobicity of IND; while the PM produced much smaller contact angle values $\left(39 \pm 2^{\circ}\right.$ after $\left.1 \mathrm{~s}\right)$, probably due to its composition L-HPC and SDS, which increases its wettability. The solid dispersion SD-1:0.12:20 had

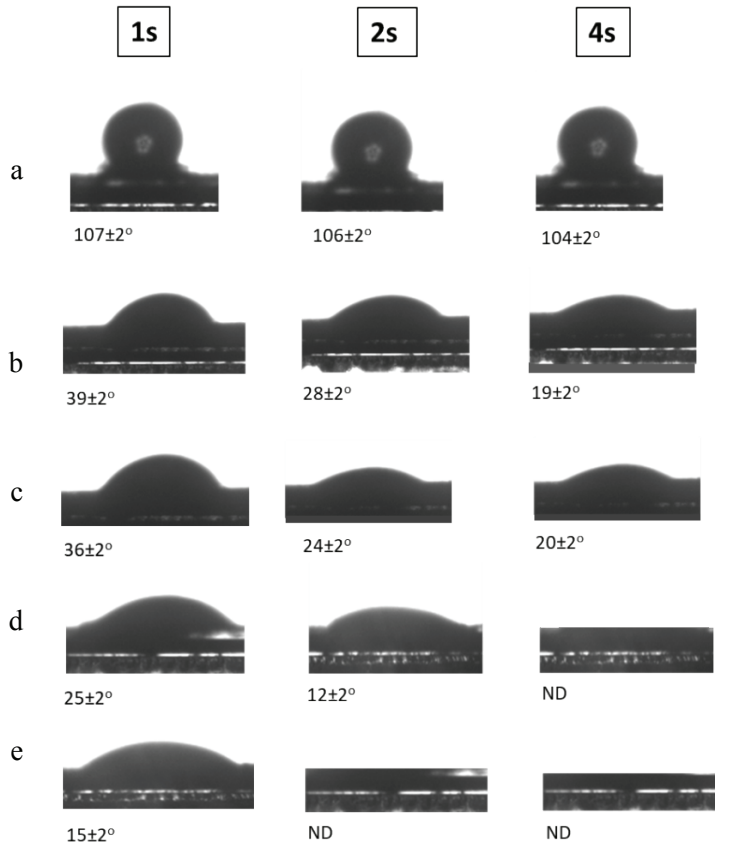

Fig. 1: Contact angle of water on IND, physical mixture and solid dispersions

Contact angle of water on a. IND, b. PM-1:0.12:20, c. SD1:0.12:0, d. SD-1:0.12:5 and e. SD-1:0.12:20. ND indicate that angle not determined 
the smallest contact angle $\left(15 \pm 2^{\circ}\right.$ after $\left.1 \mathrm{~s}\right)$, much less than the PM with the same IND:L-HPC ratio, which may be due to an increase in the wettability of the formulation after the lyophilization process. It was also observed that the solid dispersion without carrier, SD-1:0.12:0, has a contact angle of over SD-1: 0.12:5; this difference becomes greater as the L-HPC loading in the formulation increases (SD-1: 0.12:20), which may be due to the hydrophilicity of L-HPC. In view of these results, it can be concluded that L-HPC has a significant influence on the wettability of the samples.

The use of acidic medium ( $\mathrm{pH} 1.2$ ), and two intestinal media ( $\mathrm{pH} 4.5$ and $\mathrm{pH} 6.8$, respectively) is proposed to evaluate the differences in the dissolution rate of various IND formulations. These media have been used by different authors to compare various drug formulations that present substantial changes in solubility during their gastro-intestinal transit ${ }^{[29-31]}$.

The dissolution data showed low release in acidic medium (pH 1.2); less than $12.84 \pm 0.48 \%$ of IND was dissolved from the pure drug at $24 \mathrm{~h}$ and only $5.22 \pm$ $0.71 \%$ at 120 min. PM-1:0.12:20 had a similar dissolution profile, and no statistically significant differences were found between the two (fig. 2a). The addition of SDS to solid dispersions produced an increase in IND dissolution profiles, so SD-1:0.06:0 and SD-1:0.12:0 showed 28.76 $\pm 1.18 \%$ and $37.80 \pm$ $1.10 \%$ of dissolved IND respectively at $120 \mathrm{~min}$. This significant increase in the dissolution rate of the solid dispersions with a higher quantity of SDS could be attributed to the presence of SDS on the surface of the IND particles, as this ratio (IND:SDS 1:0.12) is required to produce a quicker interaction between IND and the dissolution medium ${ }^{[32]}$. The IND dissolution rate was significantly improved in all the solid dispersions at this $\mathrm{pH}$; the amount of IND dissolved (SD-1:0.12:0, SD-1:0.12:5, SD-1:0.12:10 and SD$1: 0.12: 20)$ was between $37.80 \pm 1.10$ and $41.79 \pm$ $1.72 \%$ at $120 \mathrm{~min}$, and between $41.67 \pm 2.88$ and $44.67 \pm$ $2.68 \%$ at $24 \mathrm{~h}$, respectively. No significant differences were observed among the four solid dispersions at these times. However, after short time periods $(15 \mathrm{~min})$ in this acidic medium, statistically significant differences were observed between solid dispersions with higher (SD-1:0.12:10, SD-1:0.12:20) and lower (SD-1:0.12:0, SD-1:0.12:5) L-HPC loadings. These low dissolution percentages in acidic medium had previously been observed by other authors, who reported that less than $6.2 \%$ of IND was dissolved at $24 \mathrm{~h}^{[20]}$.

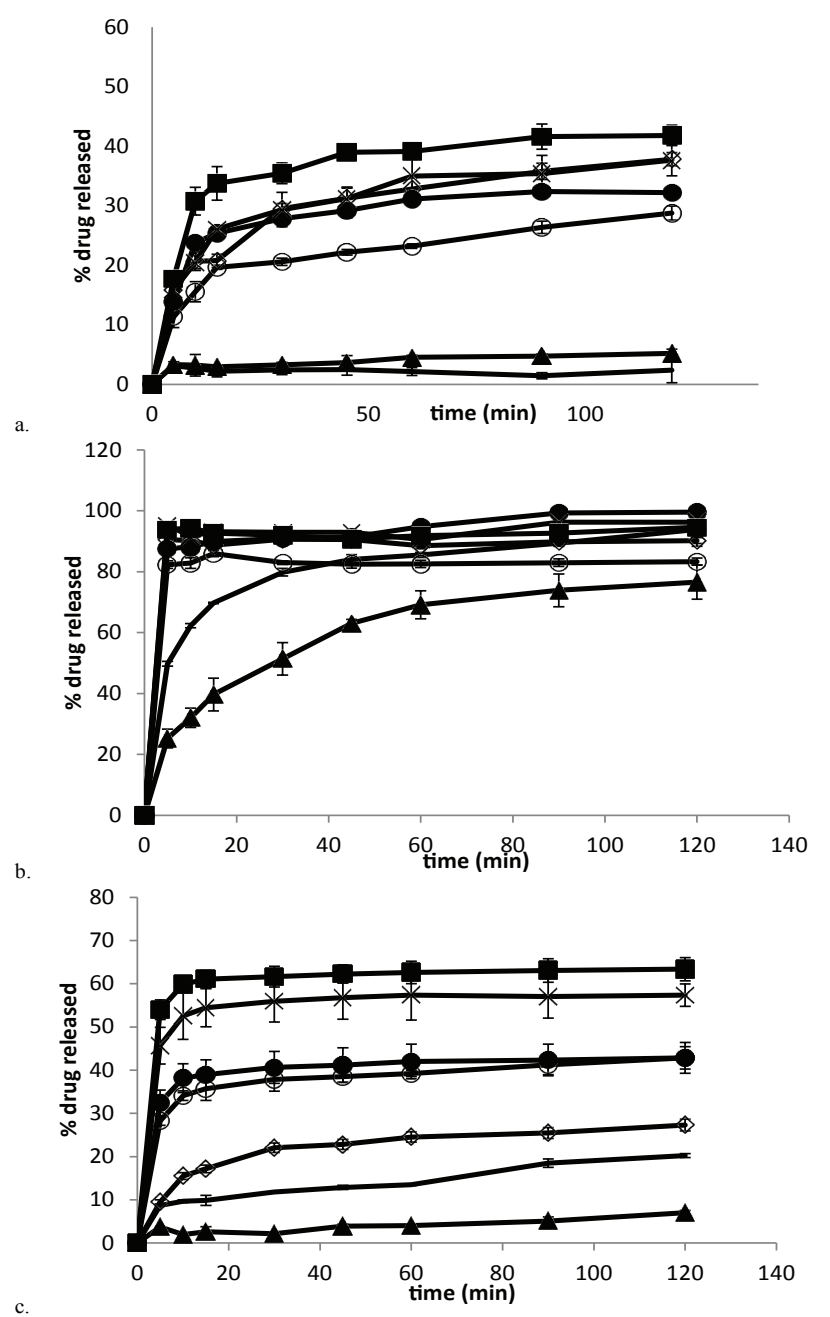

Fig. 2: Release profiles of IND formulations at $37^{\circ}$ and at different $\mathrm{pH}$ levels

Release profiles of IND formulations at $37^{\circ}$ at $\mathbf{p H}$ levels of a. 1.2, b. 6.8, and c 4.5 IND powder $(-\Delta-)$; SD-1:0.06:0 ( $-\backslash)$; SD-1:0.12:0 (—०-); SD-1:0.12:5 (-०-); SD-1:0.12:10 (一×-); SD-1:0.12:20 (—匹—) and PM-1:0.12:20 (—)

The dissolution profiles of IND powder, the PM1:0.12:20, and the different solid dispersions at $\mathrm{pH} 6.8$ (intestinal medium) are shown in fig. 2b. IND alone and PM-1:0.12:20 had the lowest dissolution rates. The amounts of IND dissolved from the drug powder compared to the amount released from PM-1:0.12:20 at $5(25.23 \pm 3.05$ and $49.75 \pm 0.80 \%$, respectively) and at $120 \mathrm{~min}(76.56 \pm 5.60$ and $93.90 \pm 5.53 \%)$ were found to be significantly different. The presence of SDS in the PM (PM-1:0.12:20) can be associated to a substantial improvement in the dissolution properties in comparison to the pure drug, and could also be seen in the percentage of IND dissolved from SD1:0.06:0 and SD-1:0.12:0. There was a significant difference between both formulations at $120 \mathrm{~min}$ $(83.33 \pm 1.16$ and $90.23 \pm 1.28 \%$, respectively), while both finally attained $100 \%$ of drug dissolved at $24 \mathrm{~h}$. 
SD-1:0.12:0 also showed a high initial burst effect at $5 \min (90.55 \pm 1.31 \%)$, which is similar to the amount of IND dissolved from the PM-1:0.12:20 at $120 \mathrm{~min}$ $(93.90 \pm 5.53 \%)$. This increase in the dissolution rate after the lyophilization process could be related to the increase in wettability observed in the contact angle measurements. The presence of higher amounts of L-HPC induces an increase in the quantity of IND dissolved from SD-1:0.12:10 and SD-1:0.12:20 at $5 \mathrm{~min}(95.21 \pm 1.16$ and $93.83 \pm 2.40 \%$, respectively) in comparison to SD-1:0.12:0 (90.55 $\pm 1.31 \%)$. Increase in the amount of IND dissolved from the solid dispersions containing higher amounts of hydrophilic carrier (L-HPC) could be attributed to a lowering of the surface tension of the medium by L-HPC, resulting in improved wetting of the hydrophobic drug surface ${ }^{[32,33]}$, according to the results obtained in the wettability measurements. At this $\mathrm{pH}$ (6.8), all solid dispersions showed higher dissolution rates, so when using this medium, no significant differences were seen between the dissolution profiles for the various solid dispersions. Other authors reported similar results using a buffer of $\mathrm{pH} 7.4$, and obtained $72.5 \pm$ $1.0 \%$ of IND dissolved from their solid dispersions at $90 \mathrm{~min}^{[34]}$. The use of buffer media with a $\mathrm{pH}$ close to the IND pKa value (4.5) leads to lower IND solubility ${ }^{[20]}$. Dissolution assays were therefore done at $\mathrm{pH} 4.5$ (fig. 2c) in order to obtain slower dissolution profiles and allow the observation of differences in the dissolution rates among the various formulations.

Fig. 2c shows the dissolution profiles of IND powder, the PM 1:20 (PM-1:0.12:20) and the various solid dispersions (SD-1:0.12:0, SD-1:0.12:5, SD-1:0.12:10, and SD-1:0.12:20) at $\mathrm{pH}$ 4.5. IND powder and PM1:0.12:20 had the poorest dissolution rates in this medium. $7.06 \pm 0.46$ and $20.26 \pm 0.80 \%$ of IND were dissolved from the pure drug and from PM-1:0.12:20 respectively at $120 \mathrm{~min}$, and $23.07 \pm 2.33$ and $29.88 \pm$ $1.25 \%$ respectively at $24 \mathrm{~h}$. As occurred at $\mathrm{pH} 6.8$, the PM showed a statistically significant improvement in the dissolution properties compared to the pure drug at $120 \mathrm{~min}$. This high amount of drug dissolved from PM$1: 0.12: 20$ could be due to the presence of SDS in this formulation, and to the wetting properties of LHPC, as seen in the wettability measurements.

IND amount dissolved from SD-1:0.12:0 at $120 \mathrm{~min}$ was $42.84 \pm 2.59 \%$. As occurred at pH 6.8, SD-1:0.12:0 significantly improves the dissolution properties of the IND powder and PM-1:0.12:20. All the solid dispersions had substantially better dissolution profiles than PM-1:0.12:20, the same as at pH 6.8. Also similar to the results at $\mathrm{pH} 6.8$, at $\mathrm{pH} 4.529 .01 \pm 0.43 \%$ of the drug was dissolved from SD-1:0.06:0 after $24 \mathrm{~h}$ and $41.67 \pm 2.88 \%$ of IND from SD-1:0.12:0 after the same amount of time. At this $\mathrm{pH}$ there is therefore a statistically significant increase in the amount of IND dissolved from solid dispersions with a higher quantity of SDS. Formulations with higher L-HPC loadings (SD-1:0.12:10 and SD-1:0.12:20) showed a statistically significant increase in their dissolution profile compared to formulations with lower L-HPC loadings. These results suggested that L-HPC itself might act as a weak solubilizer in these formulations, and might prevent drug aggregation ${ }^{[35,36]}$. These results provided evidence that all the solid dispersions were superior in achieving $100 \%$ dissolution more rapidly than the PM, i.e., over $40 \%$ of IND dissolved in the 1:0.12:10 and 1:0.12:20 solid dispersions at $120 \mathrm{~min}$.

The addition of small quantities of L-HPC to the solid dispersions did not produce any significant increments in the percentages of drug dissolved at $120 \mathrm{~min}$ (42.84 $\pm 2.59 \%$ from SD-1:0.12:0 and 42.87 $\pm 3.56 \%$ from SD-1:0.12:5, respectively). These small amounts of L-HPC might not prevent the agglomeration of IND particles during the dissolution process. However, solid dispersions with a higher quantity of L-HPC (SD1:0.12:10 and SD-1:0.12:20) increased wettability and favour a faster dissolution at the initial dissolution times, as seen in the contact angle measurements. Thus, at 5 min the amounts of IND dissolved from SD-1:0.12:5, SD-1:0.12:10 and SD-1:0.12:20 were 32.47 \pm 2.95 , $45.69 \pm 4.26$ and $54.01 \pm 2.26 \%$, respectively. Statistically significant differences observed among the different solid dispersions at this $\mathrm{pH}$ could be attributed to the poor solubility of the drug in this medium, possibly because IND solubility is $\mathrm{pH}$-dependent, at a low $\mathrm{pH}$ (1.2) it has poor solubility properties, while its solubility is greatly increased at a high $\mathrm{pH}$ (6.8). However, the use of L-HPC in solid dispersions would be justified at a duodenal $\mathrm{pH}$ of 4.5 , critical for IND, since it significantly increases the dissolution rate of formulations with high L-HPC loadings. These results also allow us to consider $\mathrm{pH} 4.5$ as a discriminating medium that can be used to distinguish the dissolution profiles of our solid dispersions with different amounts of L-HPC. The presence of L-HPC reduced the surface tension of the medium, resulting in better wetting of the hydrophobic drug surface ${ }^{[32,33]}$, according to the contact angle measurements. 
SEM was used to clarify the surface and shape characteristics of different samples (IND powder, SD0:0.12:0, SD-1:0.12:0, SD-1:0.12:5, SD-1:0.12:10, SD1:0.12:20, PM-1:0.12:20 and L-HPC). The IND powder presented heterogeneous crystals of different sizes with edges (fig. 3a). The original morphology of all the components disappeared in the solid dispersions, while the SD-1:0.12:0 observed at the same magnification (500×) (fig. 3b) had fine crystals covering their surface, possibly generated during the lyophilization process. SD-0:0.12:0 also presented acicular crystals that can be attributed to SDS and phosphate buffer components (fig. 3c). In a comparison of the PM-1:0.12:20 and the solid dispersion SD-1:0.12:20 (fig. 3d) the smooth particles of L-HPC (fig. 3e) could easily be distinguished from the heterogeneous crystals with edges, possibly related to IND, in the PM, but not in

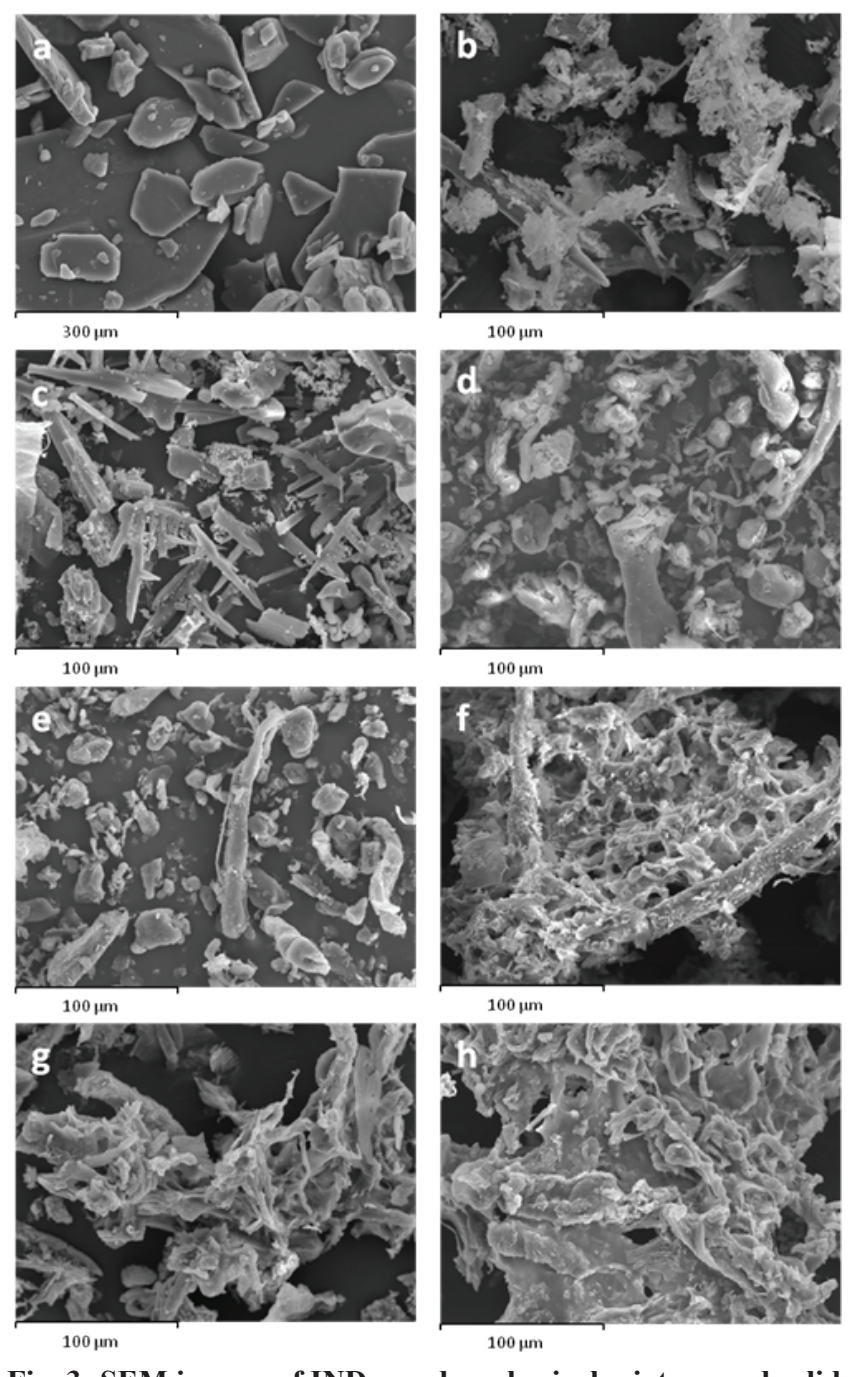

Fig. 3: SEM images of IND powder, physical mixture and solid dispersions

Scanning electron photomicrographs of a. IND powder; b. SD-1:0.12:0; c. SD-0:0.12:0; (d) PM-1:0.12:20; (e) L-HPC; (f) SD-1:0.12:5; (g) SD-1:0.12:10; (h) SD-1:0.12:20 taken at a magnification of $500 \times$ crystalline domains ${ }^{[20,25]}$. The XRPD pattern of L-HPC (fig. 4) exhibited a characteristic semi-crystalline halo diffraction pattern with low intensity values similar to pure IND between $19.8^{\circ}$ and $28.4^{\circ}(2 \theta)$, and a majority diffraction intensity at $20.1^{\circ}(2 \theta)^{[39]}$. Only one formulation excipient, boric acid (used in the 8.4 buffer), had a crystalline structure, with diffraction peaks at angles of $14.7^{\circ}, 27.7^{\circ}, 32.29^{\circ}$ and $40.3^{\circ}$ (20). In both PMs (PM-1:0.12:5 and PM-1:0.12:20) the diffraction peaks of the IND can still be seen at diffraction angles of $19.5^{\circ}$ and $21.6^{\circ}$ (20) (figs. 4a and $b$ ). The intensity of the peaks decreases with increasing amounts of L-HPC in the PM (PM-1: 0.12: 20). A reduction in drug crystallinity of nearly $55 \%$ could be seen in PM-1: $0.12: 5$, and IND crystallinity declined by approximately $88 \%$ in the PM with the highest amount of LHPC (PM-1:0.12:20). This result can be attributed to the dilution effect with higher amounts of carrier. As expected, the PM-1: 0.12: 20 showed higher intensity values for the semi-crystalline halo of the carrier (between $19.8^{\circ}$ and $28.4^{\circ}(2 \theta)$ ). The high L-HPC loadings in this PM justified the presence of this halo (fig. 4b). Moreover, SD-1:0.12:0 (fig. 4) showed practically the same peaks at $15.9^{\circ}$, $28.3^{\circ}, 32.3^{\circ}$ and $40.4^{\circ}(2 \theta)$, related to the boric acid, component of $\mathrm{pH} 8.4$ buffer. The diffraction peaks of the IND could not be seen in this sample. The absence of crystallinity in the IND in this formulation could be caused by the freeze-drying process ${ }^{[40]}$. This decrease the solid dispersion. In contrast, solid dispersions with
different L-HPC loadings (SD-1:0.12:5, SD-1:0.12:10 and SD-1:0.12:20) resembled a matrix (figs. $3 \mathrm{f}$, $g$ and $\mathrm{h}$, respectively). In these L-HPC solid dispersions, the higher the loading of L-HPC, the lower the porosity
of the matrix. These results demonstrated that IND was dispersed homogeneously in the L-HPC polymer matrix of the solid dispersions, where it could possibly Fig. 4 shows the XRPD patterns of the pure IND powder, SD-1:0.12:0, SD-1:0.12:10, SD-1:0.12:20,
PM-1:0.12:20, PM-1:0.12:5 and L-HPC. IND powder has low-intensity values in its diffraction peaks, typical of a substance with low crystallinity (see fig. 4). The crystal structure of the pure drug with low intensity
peaks at angles of $11.6^{\circ}, 17.2^{\circ}, 19.5^{\circ}, 21.6^{\circ}$ and $29.3^{\circ}$ (20) almost matches polymorph $\gamma$, as reported by other authors $^{[38]}$. This low drug crystallinity may be related to the heterogeneous shape of its crystals with edges and different sizes observed in SEM studies (fig. 3). 
in crystallinity after the freeze-drying process could be related to improvements in the dissolution profiles, as observed in the dissolution assays. Solid dispersions with different proportions of L-HPC (fig. 4) present a similar result to SD-1:0.12:0, and do not show any of the peaks attributed to IND.

Both formulations, SD-1:0.12:10 and SD-1:0.12:20, presented a semi-crystalline halo diffraction pattern from L-HPC, with lower intensities at $20.1^{\circ}$ (20). The lower crystallinity in the halo attributed to the carrier for the solid dispersions may confirm that the freeze-drying process decreases the crystallinity in these formulations. The inferior sensitivity of the XRPD method also makes it difficult to determine the crystallinity peaks of samples with low-intensity values such as IND. Similar results were obtained by other authors $^{[41]}$. DSC studies will be necessary to complete these studies on the decline in crystallinity.

Fig. 5 shows thermograms for pure IND; SD-1:0.12:0, SD-1:0.12:10, SD-1:0.12:20, PM-1:0.12:20 and L-HPC. IND powder (fig. 5a) has an endothermic peak at $160.99^{\circ}$ with low crystallinity $(116.05 \mathrm{~J} / \mathrm{g})^{[42]}$. Fig. 5 shows a small endothermic peak for L-HPC at $158.31^{\circ}$ (onset $157.14^{\circ}$ ); the low enthalpy of fusion of this peak $(52.34 \mathrm{~mJ} / \mathrm{g})$ was related to its semicrystalline structure $^{[43]}$. Among the other formulation excipients, only boric acid, used in the $\mathrm{pH} 8.4$ buffer, had a crystalline structure with two endothermic peaks at $167.22^{\circ}$ (minor) and $170.31^{\circ}$ (major), in addition to characteristic changes in the baseline at higher temperatures; while SDS and the other excipients had no endothermic peaks characteristic of crystalline substances.
Two thermal events occur in PM-1: 0.12: 20 (fig. 5b), showing a minor peak at $144.84^{\circ}$ and a major peak at $168.22^{\circ}$. The IND melting peak in the curve for this PM underwent a shift to a lower temperature $\left(144.84^{\circ}\right)$, while the melting peak for L-HPC and boric acid shifted to a higher temperature $\left(168.22^{\circ}\right)$. This dip in the IND melting point is a common effect in multicomponent mixtures and is considered a positive interaction with the L-HPC and other crystalline formulation excipients ${ }^{[44-46]}$. The sharper peak at $168.22^{\circ}$ observed in PM-1:0.12:20 can be attributed to a combination of two of the components in the formulation: the first is L-HPC, which may have shifted to higher temperatures (from $158.31^{\circ}$ to $\left.168.22^{\circ}\right)^{[43]}$; and the second is boric acid, a highly crystalline substance used in the buffer. The presence of high amounts of L-HPC produces a single endothermic peak at $168^{\circ}$ where boric acid is included. A similar process of inclusion in a single peak has been observed by different authors ${ }^{[47]}$.

In contrast, in the SD-1:0.12:0 thermogram (fig 5c), only a slight amorphous halo was observed between $154-166^{\circ}$, which may be related to a decrease in IND crystallinity, possibly due to the lyophilization process. No peaks attributed to buffer components were observed in this lyophilized sample. IND:SDS:L-HPC solid dispersions (SD-1:0.12:10 and SD-1:0.12:20) showed both an incipient decrease to lower temperatures and also a widening in the amorphous halo (between 126$162^{\circ}$ for SD-1:0.12:10 and $122-158^{\circ}$ for SD-1:0.12:20, respectively, figs. $5 \mathrm{~d}$ and e). The onset of the amorphous halo of the IND decreases as the proportion of L-HPC in the sample increases. These results confirm the

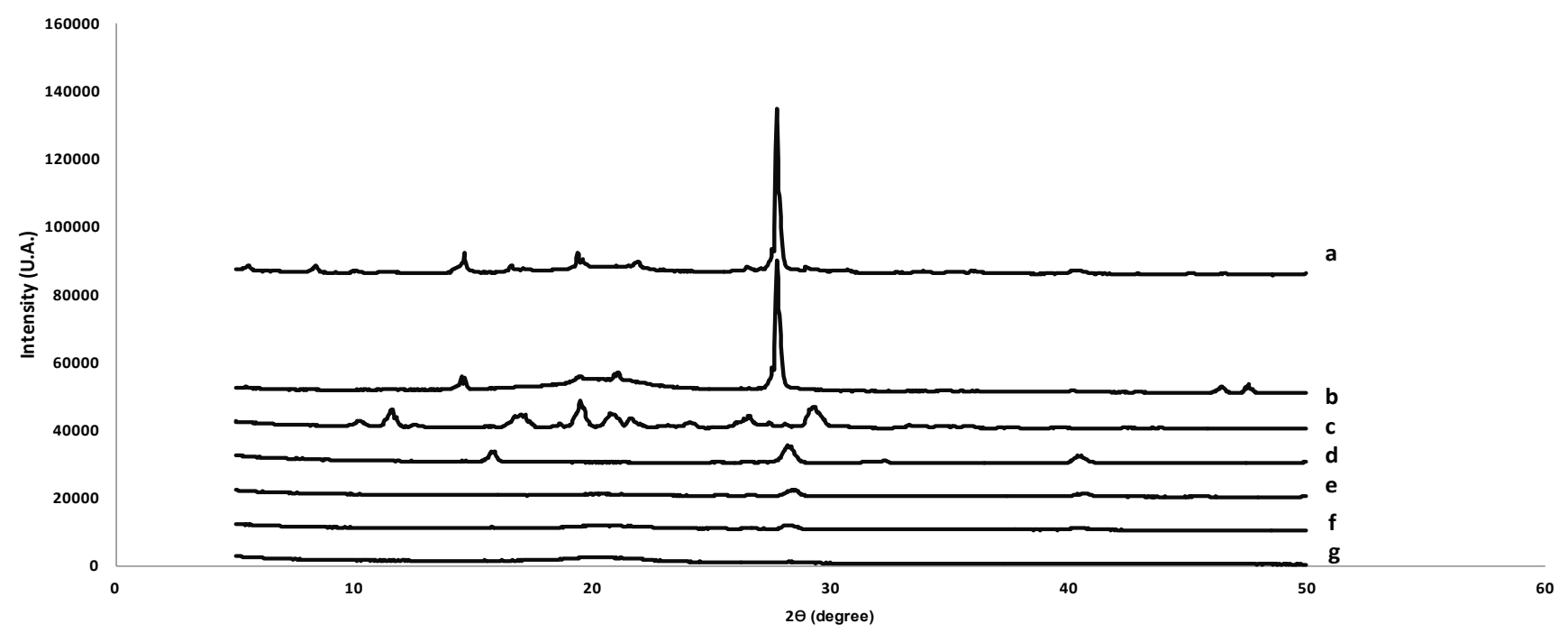

Fig. 4: X-ray powder diffraction scans (a) PM-1:0.12:5; (b) PM-1:0.12:20; (c) pure IND; (d) SD-1:0.12:0; (e) SD-1:0.12:10; (f) SD-1:0.12:20 and (g) L-HPC 


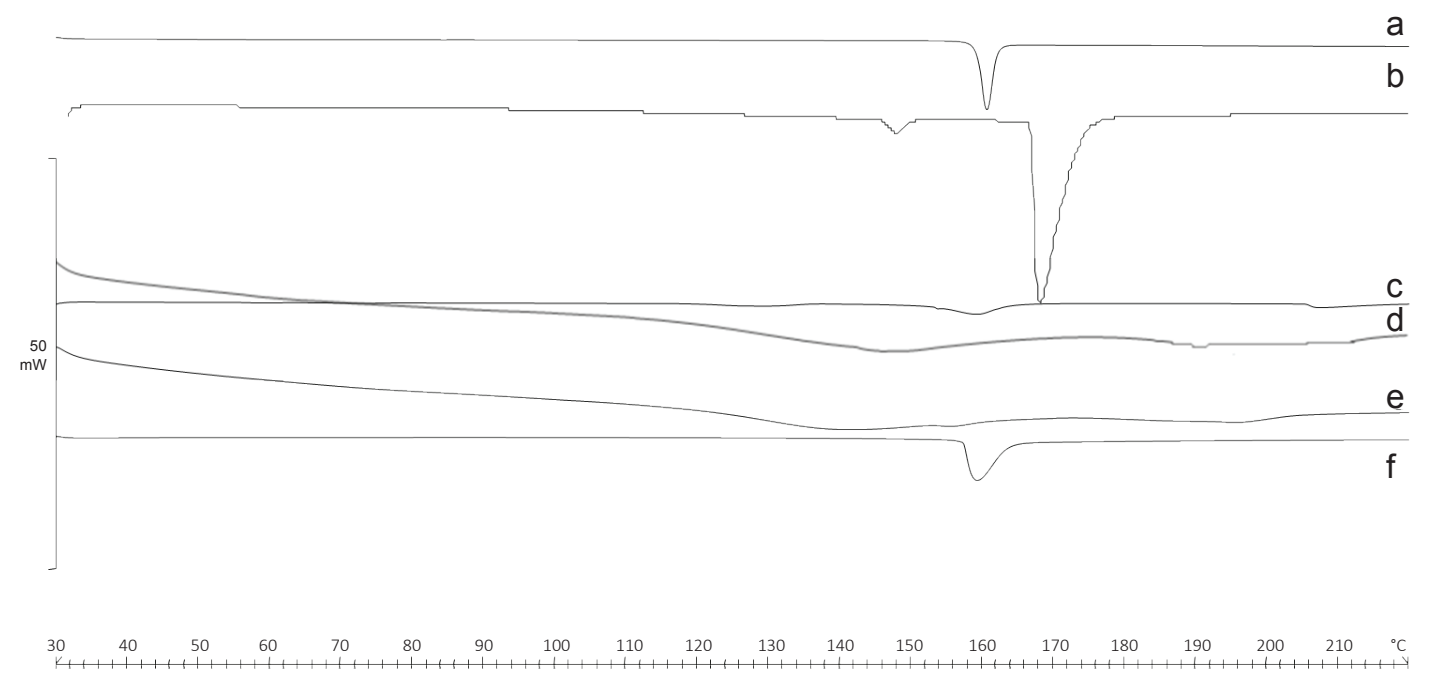

Fig. 5: DSC thermograms

(a) IND; (b) PM-1:0.12:20; (c) SD-1:0.12:0; (d) SD-1:0.12:10; (e) SD-1:0.12:20; and (f) L-HPC

existence of a positive interaction between the IND and the L-HPC excipient in lyophilized samples.

The amorphous character of IND in the solid dispersions observed in the DSC studies confirmed the hypothesis that amorphous species of IND are being formed in the XRPD studies. Solid dispersions could modify drug crystallinity and represent a suitable modification for improving its dissolution profiles. These results were also confirmed in the dissolution assays, where improvements were observed in the dissolution profiles of the various solid dispersions compared to the IND powder and PM-1:0.12:20. Amorphous substances generally have a higher solubility and dissolution rate than their corresponding thermodynamically stable crystalline forms, as their internal bonding forces are weak $^{[48]}$.

In summary, IND:SDS:L-HPC solid dispersions improve the dissolution rate of IND. Wettability measurements, SEM, XRPD and DSC techniques were used to study the reasons for this, and suggest that an increase in the IND dissolution rate is caused by a reduction in drug crystallinity related to the freezedrying process and the amount of L-HPC added to the formulations. The addition of a hydrophilic carrier altered the surface morphology of the formulations and also increased their wetting effect. The formation of a less porous L-HPC matrix could be observed by SEM, as the proportion of polymer increased. From all of the above, it can be deduced that all these factors could cause an important increase in the IND dissolution rate.

\section{Acknowledgements:}

The authors would like to thank the Department of
Biomedical Sciences, Alcalá University, Spain, and the Department of Physical Chemistry, Faculty of Chemical Sciences, Madrid Complutense University, Spain for their support.

\section{Conflict of interest:}

No conflict of interest between any of the authors.

\section{REFERENCES}

1. Shristi B, Neha J, Indu BP, Rajesh G. A review on some Indian medicinal plants for antiulcer activity. J Sci Res Pharm 2012;1:6-9.

2. Suleyman H, Albayrak A, Bilici M, Cadirci E, Halici Z. Different mechanisms in formation and prevention of indomethacin-induced gastric ulcer. Inflammation 2010;33(4):224-34.

3. Lichtenberger LM, Phan T, Fang D, Dial EJ. Chemoprevention with phosphatidylcholine non-steroidal anti-inflammatory drugs in vivo and in vitro. Oncol Lett 2018;15(5):6688-94.

4. Grau MV, Baron JA, Langholz B, Karagas M, Greenberg ER, Stukel TA, et al. Effect of NSAIDs on the recurrence of nonmelanoma skin cancer. Int J Cancer 2006;119:682-6.

5. Somasundaram R, Herlyn M. Indomethacin to the rescue of TRAIL-resistant melanomas. J Invest Dermatol 2014;134(5):1198-9.

6. Martínez LM, Videa M, López-Silva T, Castro S, Caballero A, Lara-Díaz VJ, et al. Two-phase amorphous-amorphous solid drug dispersion with enhanced stability, solubility and bioavailability resulting from ultrasonic dispersion of an immiscible system. Eur J Pharm Biopharm 2017;119:243-52.

7. fda.gov [Internet]. FDA: U.S. Food and Drug Administration; [cited 2018 Sep 27]. Available from: https://www.fda.gov/.

8. Vo CL, Park C, Lee BJ. Current trends and future perspectives of solid dispersions containing poorly water-soluble drugs. Eur J Pharm Biopharm 2013;85:799-813.

9. Berge SM, Bighley LD, Monkhouse DC. Pharmaceutical salts. J Pharm Sci 1977;66(1):1-19.

10. Mueller EA, Kovarik JM, van Bree JB, Tetzloff W, 
Grevel J, Kutz K. Improved dose linearity of cyclosporine pharmacokinetics from a microemulsion formulation. Pharm Res 1994;11(2):301-4.

11. Muller RH, Peters K. Nanosuspensions for the formulation of poorly soluble drugs: I. Preparation by a size-reduction technique. Int J Pharm 1998;160:229-37.

12. Jones M, Leroux J. Polymeric micelles - a new generation of colloidal drug carriers. Eur J Pharm Biopharm 1999;48(2):101-11.

13. Fatouros DG, Deen GR, Arleth L, Bergenstahl B, Nielsen FS, Pedersen JS, et al. Structural development of self-nano emulsifying drug delivery systems (SNEDDS) during in vitro lipid digestion monitored by small-angle Xray scattering. Pharm Res 2007;24(10):1844-53.

14. Roy S, Pal M, Gupta BK. Indomethacin-loaded microspheres: design and preparation by a multiple- emulsification technique and their in vitro evaluation. Pharm Res 1992;9(9):1132-6.

15. Rabinow BE. Nanosuspensions in drug delivery. Nat Rev Drug Discov 2004;3(9):785-96.

16. Brewster ME, Loftsson T. Cyclodextrins as pharmaceutical solubilizers. Adv Drug Deliv Rev 2007;59(7):645-66.

17. Rautio J, Kumpulainen H, Heimbach T, Oliyai R, Oh D, Jarvinen T, et al. Prodrugs: design and clinical applications. Nat Rev Drug Discovery 2008;7(3):255-70.

18. Potta SG, Minemi S, Nukala RK, Peinado C, Lamprou DA, Urquhart A, et al. Development of solid lipid nanoparticles for enhanced solubility of poorly soluble drugs. J Biomed Nanotechnol 2010;6(6):634-40.

19. Sekiguchi K, Obi N, Ueda Y. Studies on absorption of eutectic mixture. I. A comparison of the behavior of eutectic mixture of sulfathiazole and that of ordinary sulfathiazole in man. Chem Pharm Bull 1961;9:866-72.

20. Saeedi M, Akbari J, Morteza-Semnani K, Enayati-Fard R, SarReshteh-Dar S, Soleymani A. Enhancement of dissolution rate of indomethacin using liquisolid compacts. Iran J Pharm Res 2011;10(1):25-34.

21. Baghel S, Cathcart H, O'Reilly NJ. Investigation into the solid-state properties and dissolution profile of spray-dried ternary amorphous solid dispersions: a rational step toward the design and development of a multicomponent amorphous system. Mol Pharm 2018;15(9):3796-812.

22. Shirai Y, Sogo K, Fujioka H, Nakamura Y. Role of lowsubstituted hydroxypropylcellulose in dissolution and bioavailability of novel fine granule system for masking bitter taste Biol Pharm Bull 1994;17:427-31.

23. Halder S, Tabata A, Seto Y, Sato H, Onoue S. Amorphous solid dispersions of carvedilol along with $\mathrm{pH}$-modifiers improved pharmacokinetic properties under hypochlorhydoria. Biopharm Drug Dispos 2018;39(4):232-42.

24. Ponnammal P, Kanaujia P, Yani Y, Ng WK, Tan RBH. Orally disintegrating tablets containing melt extruded amorphous solid dispersion of tacrolimus for dissolution enhancement. Pharmaceutics 2018;10(1):E35.

25. Sarode AL, Malekar SA, Cote C, Worthen DR. Hydroxypropyl cellulose stabilizes amorphous solid dispersions of the poorly water soluble drug felodipine. Carbohydr Polym 2014;112:512-9.

26. de la Torre-Iglesias PM, García-Rodríguez JJ, Torrado-Durán G, Torrado-Durán S, Torrado-Santiago S, Bolás-Fernández F. Enhanced bioavailability and anthelmintic efficacy of mebendazole in redispersible microparticles with lowsubstituted hydroxypropylcellulose. Drug Des Devel Ther 2014;8:1467-79.

27. Mesnukul A, Yodkhum K, Mahadlek J, Phaechamud T. Characterization of indomethacin release from polyethylene glycol tablet fabricated with mold technique. Indian J Pharm Sci 2010;72(1):92-100.

28. Dahlberg C, Millqvist-Fureby A, Schuleit M, Furó I. Polymerdrug interactions and wetting of solid dispersions. Eur J Pharm Sci 2010;39(1-3):125-33.

29. El-Sabawi D, Hamdan II. Improvement of dissolution rate of gliclazide through sodium salt formation. Dissolut Technol 2014;21(4):49-55.

30. Stuart AV, Zuo J, Löbenberg R. Investigating the dissolution profiles of amoxicillin, metronidazole, and zidovudine formulations used in Trinidad and Tobago, West Indies. AAPS PharmSciTech 2014;15(5):1060-9.

31. Oishi TS, Haque MA, Dewan I, Islam SMA. Comparative in vitro dissolution study of some ciprofloxacin generic tablets under biowaiver conditions by RP-HPLC. Int J Pharm Sci Res 2011;2(12):3129-35.

32. García-Herrero V, Torrado C, García-Rodríguez JJ, LópezSánchez A, Torrado S, Torrado-Santiago S. Improvement of the surface hydrophilic properties of naproxen particles with addition of hydroxypropylmethyl cellulose and sodium dodecyl sulphate: In vitro and in vivo studies. Int J Pharm 2017;529(1-2):381-90.

33. Ambike AA, Mahadik KR, Paradkar A. Stability study of amorphous valdecoxib. Int J Pharm 2004;282(1-2):151-62.

34. El-Badry M, Fetih G, Fathy M. Improvement of solubility and dissolution rate of indomethacin by solid dispersions in Gelucire 50/13 and PEG4000. Saudi Pharm J 2009;17(3):217-25.

35. Tantishaiyakul V, Kaewnopparat N, Ingkatawornwong S. Properties of solid dispersions of piroxicam in polyvinylpyrrolidone K-30. Int J Pharm 1999;181(2):143-51.

36. Kawabata Y, Yamamoto K, Debari K, Onoue S, Yamada S. Novel crystalline solid dispersion of tranilast with high photostability and improved oral bioavailability. Eur J Pharm Sci 2010;39(4):256-62.

37. Yamashita K, Nakate T, Okimoto K, Ohike A, Tokunaga $\mathrm{Y}$, Ibuki R, et al. Establishment of new preparation method for solid dispersion formulation of tacrolimus. Int J Pharm 2003;267(1-2):79-91.

38. Semjonov K, Kogermann K, Laidmäe I, Antikainen O, Strachan CJ, Ehlers H, et al. The formation and physical stability of two-phase solid dispersion systems of indomethacin in supercooled molten mixtures with different matrix formers. Eur J Pharm Sci 2016;97:237-46.

39. Kiyose M, Yamamoto E, Yamane C, Midorikawa T, Takahashi T. Structure and Properties of Low-Substituted Hydroxypropylcellulose Films and Fibers Regenerated from Aqueous Sodium Hydroxide Solution. Polym J 2007;39:703-11.

40. Pralhad T, Rajendrakumar K. Study of freeze-dried quercetincyclodextrin binary systems by DSC, FT-IR, X-ray diffraction and SEM analysis. J Pharm Biomed Anal 2004;34(2):333-9.

41. García-Rodríguez JJ, de la Torre-Iglesias PM, Vegas-Sánchez $\mathrm{MC}$, Torrado-Durán S, Bolás-Fernández F, Torrado-Santiago S. Changed crystallinity of mebendazole solid dispersion: Improved anthelmintic activity. Int J Pharm 2011;403(12):23-8. 
42. Rojas-Oviedo I, Retchkiman-Corona B, Quirino-Barreda CT, Cárdenas J, Schabes-Retchkiman PS. Solubility enhancement of a poorly water soluble drug by forming solid dispersions using mechanochemical activation. Indian $\mathrm{J}$ Pharm Sci 2012;74(6):505-11.

43. Palmeiro Palmeiro-Roldán R, Fonseca-Berzal C, GómezBarrio A, Arán VJ, Escario JA, Torrado-Durán $\mathrm{S}$, et al. Development of novel benznidazole formulations: physicochemical characterization and in vivo evaluation on parasitemia reduction in Chagas disease. Int J Pharm 2014;472(1-2):110-7.

44. Gómez-Burgaz M, Torrado G, Torrado S. Characterization and superficial transformations on mini-matrices made of interpolymer complexes of chitosan and carboxymethylcellulose during in vitro clarithromycin release. Eur J Pharm Biopharm 2009;73(1):130-9.
45. Löbmann K, Wohlert J, Müllertz A, Wågberg L, Svagan AJ. Indomethacin: Cellulose nanopaper and nanofoam for patient-tailored drug delivery. Adv Mater Interfaces 2017;9(4):1600655.

46. Bannow J, Benjamins JW, Wohlert J, Löbmann K, Svagan AJ. Solid nanofoams based on cellulose nanofibers and indomethacinthe effect of processing parameters and drug content on material structure. Int J Pharm 2017;526(1-2):291-9.

47. Pani NR, Nath LK, Acharya S, Bhuniya B. Application of DSC, IST, and FTIR study in the compatibility testing of nateglinide with different pharmaceutical excipients. J Therm Anal Calorim 2012;108:219-26.

48. Sun Y, Zhu L, Wu T, Cai T, Gunn EM, Yu L. Stability of amorphous pharmaceutical solids: crystal growth mechanisms and effect of polymer additives. AAPS J 2012;14(3):380-8. 